\title{
BMJ Open Protocol for the specialist supervised individualised multifactorial treatment of new clinically diagnosed type 2 diabetes in general practice (IDA): a prospective controlled multicentre open-label intervention study
}

\author{
Jacob Volmer Stidsen, ${ }^{1}$ Jens Steen Nielsen, ${ }^{1}$ Jan Erik Henriksen, \\ Søren Gunnar Friborg, ${ }^{1}$ Reimar Wernich Thomsen, ${ }^{2}$ Thomas Bastholm Olesen, ${ }^{1}$ \\ Michael Hecht Olsen, ${ }^{1}$ Henning Beck-Nielsen ${ }^{1}$
}

To cite: Stidsen JV, Nielsen JS, Henriksen JE, et al. Protocol for the specialist supervised individualised multifactorial treatment of new clinically diagnosed type 2 diabetes in general practice (IDA): a prospective controlled multicentre open-label intervention study. BMJ Open 2017;7:e017493. doi:10.1136/ bmjopen-2017-017493

- Prepublication history and additional material for this paper are available online. To view these files, please visit the journal online (http://dx.doi. org/10.1136/bmjopen-2017017493).

Received 26 April 2017 Revised 7 September 2017 Accepted 12 October 2017

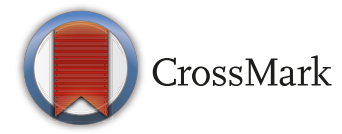

1Department of Endocrinology, Diabetes Research Centre, Centre for Individualized Medicine in Arterial Diseases, Odense University Hospital, Odense, Denmark

${ }^{2}$ Department of Clinical Epidemiology, Aarhus University Hospital, Aarhus, Denmark

Correspondence to

Henning Beck-Nielsen;

henning.beck-nielsen@rsyd.dk

\section{ABSTRACT}

Introduction We present the protocol for a multifactorial intervention study designed to test whether individualised treatment, based on pathophysiological phenotyping and individualised treatment goals, improves type 2 diabetes (T2D) outcomes.

Methods and analysis We will conduct a prospective controlled multicentre open-label intervention study, drawing on the longitudinal cohort of the Danish Centre for Strategic Research in Type 2 Diabetes (DD2). New clinically diagnosed patients with $\mathrm{T} 2 \mathrm{D}$ in the intervention group will be assigned to receive individualised treatment by their general practitioner. Intervention patients will be compared with a matched control cohort of DD2 patients receiving routine clinical care. Among intervention patients, we will first do pathophysiological phenotyping to classify patients into WHO-defined T2D or other specific types of diabetes (monogenic diabetes, secondary diabetes etc). Patients with WHO-defined T2D will then be further subcharacterised by their beta-cell function (BCF) and insulin sensitivity (IS), using the revised homeostatic assessment model, as having either insulinopaenic T2D (high IS and low BCF), classical T2D (low IS and low BCF) or hyperinsulinaemic T2D (low IS and high BCF). For each subtype, a specific treatment algorithm will target the primary pathophysiological defect. Similarly, antihypertensive treatment will be targeted at the specific underlying pathophysiology, characterised by impedance cardiography (relative importance of vascular resistance, intravascular volume and cardiac inotropy). All treatment goals will be based on individual patient assessment of expected positive versus adverse effects. Web-based and face-to-face individualised lifestyle intervention will also be implemented to empower patients to make a sustainable improvement in daily physical activity and to change to a low-carbohydrate diet.

Ethics and dissemination The study will use well-known pharmacological agents according to their labels; patient safety is therefore considered high. Study results will be published in international peer-reviewed journals.
Strengths and limitations of this study

- The 'Individualised treatment of newly clinical diagnosed T2D in general practice' study is a nationwide intervention study in primary care, based on a close cooperation between hospital-based diabetes specialists and general practitioners.

- Endocrinological assessment of pathophysiological phenotypes will form the basis for individual treatment algorithms, made readily available to primary healthcare providers.

- The study will clarify if an individualised approach to the pharmacological and lifestyle treatment of T2D with individualised treatment goals is associated with a range of improved hard outcomes in everyday clinical practice, including microvascular and macrovascular complications and death.

- The study is not randomised, and potential differences in prognostic factors between intervention and control patients need to be addressed by rigorous statistical methods.

- Existing high-quality healthcare registries will be used for assessment of outcomes, rather than primary adjudication of endpoints.

Trial registration number NCT02015130; Pre-results.

\section{INTRODUCTION}

Importance of individualised glycaemic control

Although current advances in type 2 diabetes (T2D) treatment have reduced mortality $^{1}$ and possibly complications ${ }^{2}$ among patients with T2D, they still suffer excess mortality compared with people without diabetes. ${ }^{3}$ Poor glycaemic control has been linked to cardiovascular morbidity, even below the threshold 
for diabetes, ${ }^{4}$ although increased mortality is also seen in the lowest 10 th percentile of haemoglobin A1c (HbA1c) values. ${ }^{5}$ This has led to several trials testing intensive glucose-lowering against moderate glucose-lowering strategies. ${ }^{6-8}$ Their results have been inconclusive, and a meta-analysis of trials of intensive glucose lowering found no effect on mortality (risk ratio (RR) $1.04,0.91-1.19$ ) or cardiovascular mortality (RR 1.11, 0.86-1.43). A significant effect (RR 0.85, 0.74-0.96) was observed for non-fatal myocardial infarction, although in analyses restricted to high-quality studies, there was no favourable effect for any myocardial infarction (RR 1.34, 0.77-2.35). In addition, a potential effect was observed for new or worsening retinopathy (RR $0.85,0.71-1.03) .{ }^{9}{ }^{10} \mathrm{On}$ the other hand, intensive glucose control was associated with a significant increase in severe hypoglycaemic events (RR 2.33, 1.62-3.36). ${ }^{9}$ The analysed trials are heterogeneous with respect to diabetes duration among included patients and achieved HbA1c. In the UK Prospective Diabetes Study, intensive glucose lowering to an HbAlc of $7.0 \%$ in newly diagnosed T2D was associated with reduced all-cause mortality, ${ }^{11-13}$ and this has recently been confirmed in an observational study design. ${ }^{14}$ Intensive glucose lowering of HbAlc to $6.4 \%$ in the The Action to Control Cardiovascular Risk in Diabetes (ACCORD) trial among patients with longer duration of diabetes was associated with an increased risk of all-cause and cardiovascular mortality. ${ }^{6}{ }^{15}$ Post hoc analyses of The Action in Diabetes and Vascular Disease: Preterax and Diamicron Modified Release Controlled Evaluation (ADVANCE) study, which sought to determine the effect on cardiovascular disease (CVD) of intensive glucose-lowering compared with standard glucose-lowering treatment, have shown that patients with severe hypoglycaemic events have a higher incidence of microvascular and macrovascular events as well as mortality. ${ }^{16}$ Together, these results indicate the necessity for an individualised approach, with differentiated goals for glycaemic control. A tight glycaemic goal of $48 \mathrm{mmol} / \mathrm{mol}$ seems relevant for many patients with newly diagnosed T2D, while patients with former CVD, neuropathy or high risk of hypoglycaemic events arguably could aim for an HbAlc below $58 \mathrm{mmol} /$ mol. ${ }^{17}$ Frail patients should aim for relief of hyperglycaemic symptoms and treatment should confer a very low hypoglycaemic risk. ${ }^{17}$

Non-vascular outcomes in T2D become increasingly important these years. The incidence of cancer overall and of several specific cancers is substantially increased in patients with T2D compared with persons without diabetes, ${ }^{18} 19$ and also mortality from cancer is increased. ${ }^{20}$ Whether specific glucose-lowering therapies are associated with increased or reduced risk of cancer remains uncertain. A meta-analysis has reported a possibly reduced cancer risk with metformin and thiazolidinedione therapy, and an increased risk with insulin, sulfonylurea and alpha glucosidase inhibitor use. When the meta-analysis was restricted to randomised controlled trials (RCTs), these associations could not be confirmed, with the limitation that most RCTs are too short to properly elucidate cancer risk and have heterogeneous comparators. ${ }^{21}$ These uncertainties highlight the need for long-term evaluation of therapy not only with regard to microvascular and macrovascular disease but also with respect to cancer risk.

\section{Improved glycaemic control through better pathophysiological phenotyping}

Diabetes is classified into type 1 diabetes, T2D, other specific types of diabetes and gestational diabetes. ${ }^{22}$ It has become increasingly clear that diabetes is a more heterogeneous disease. ${ }^{23}$ Data from the Danish Centre for Strategic Research in Type 2 Diabetes (DD2) show that in clinical practice, patients with other specific types of diabetes than T2D (for example, glucocorticoid-induced diabetes, late autoimmune diabetes of the adult or secondary diabetes) are often misclassified as patients with classical WHO-defined T2D. As the pathophysiology of other specific types of diabetes is potentially different from classical WHO-defined T2D, ${ }^{24}$ poor glycaemic control could be a consequence if the given treatment does not address the underlying pathophysiological defect. Moreover, DD2 data also show that the classical WHO-defined T2D population is heterogeneous and may be further classified according to pathophysiological phenotypes, with potential implications for appropriate glucose-lowering treatment. ${ }^{25}$ In addition, ethnic differences in the pathophysiology of T2D have been reported, ${ }^{26} 27$ underlining the importance of both interethnical and intraethnical heterogeneity in T2D.

\section{Importance of individualised blood pressure control}

Elevated blood pressure in patients with T2D is associated with cardiovascular death, starting with a systolic blood pressure of $120 \mathrm{~mm} \mathrm{Hg}{ }^{28}$ A recent meta-analysis concluded that when systolic office blood pressure was below $140 \mathrm{~mm} \mathrm{Hg}$, further reduction in blood pressure was associated with increased risk of cardiovascular death in patients with diabetes. ${ }^{29}$ However, even under optimal conditions, blood pressure control is very difficult to achieve, with only $50 \%$ of patients reaching a systolic blood pressure below $140 \mathrm{~mm} \mathrm{Hg}{ }^{30}$ Impedance cardiography has been shown to increase the proportion of patients who achieve blood pressure control. ${ }^{31} \mathrm{~A}$ recent study in a specialised hypertension clinic could not replicate this finding, although the incidence of adverse events was significantly reduced in patients in the impedance group ${ }^{32}$ Impedance cardiography offers an assessment of cardiac contractility, vascular resistance and intravascular volume. In the 'Individualised treatment of newly clinical diagnosed T2D in general practice' (IDA) study, these estimates will be used to guide selection of antihypertensive treatment in order to obtain better blood pressure control and to reduce side effects. 


\section{Importance of individualised lifestyle changes}

Lifestyle changes are the first-choice treatment for patients with newly diagnosed T2D. However, such changes are often difficult to implement and also costly if they need to be supervised. Promoting individualised lifestyle changes will be an important part of this study. Our aim is to provide evidence-based lifestyle interventions that are feasible to implement on an everyday basis. We hope to empower patients to implement changes in their everyday life via face-to-face consultations and novel individualised supportive E-health solutions. We plan to identify and describe patients who will benefit clinically from the E-health solutions being offered and to use this knowledge for large-scale implementation of individualised E-health technology in daily clinical practice.

\section{Importance of multifactorial management of T2D}

The Steno 2 study underlined the importance of multifactorial intervention in longer-standing T2D with a marked and durable reduction in morbidity and mortality associated with multifactorial intervention. ${ }^{1}$ A multifactorial approach is also emphasised in the current diabetes guidelines. ${ }^{173}$ In the current study, we therefore aim to develop specific individualised approaches to the various components of a multifactorial intervention.

\section{Importance of diabetes management in general practice}

Primary healthcare providers have an integrated knowledge of the medical history, social status and family relationships of their patients, together with a general knowledge of treatment. IDA is designed to integrate specialist knowledge and examinations into the treatment of patients in primary care. Endocrinological assessment of pathophysiological phenotypes will form the basis for individual treatment algorithms, made readily available to primary healthcare providers.

\section{HYPOTHESIS}

We hypothesise that individualised treatment based on pathophysiological traits and a new guidance strategy will improve glycaemic and blood pressure regulation and reduce complications in patients with clinically diagnosed T2D compared with outcomes under current guidelines.

Table 1 Data sources

\begin{tabular}{|c|c|c|c|}
\hline Variables & Source & Intervention & Control \\
\hline DNA samples & DD2 biobank & Yes & Yes \\
\hline $\begin{array}{l}\text { Baseline clinical variables (smoking, physical exercise, } \\
\text { alcohol consumption) }\end{array}$ & DD2 interview & Yes & Yes \\
\hline $\begin{array}{l}\text { Longitudinal clinical measures (BMI, } \\
\text { waist circumference, blood pressure, smoking) }\end{array}$ & Danish Diabetes Database for Adults & Yes & Yes \\
\hline Medical history at baseline (hospital contact history) & The Danish National Patient Register & Yes & Yes \\
\hline Medication during the study & $\begin{array}{l}\text { The Danish National Prescription } \\
\text { Registry }\end{array}$ & Yes & Yes \\
\hline Intended medication & $\begin{array}{l}\text { National electronic medicine chart } \\
\text { Fælles medicinkort (FMK) }\end{array}$ & Yes & Yes \\
\hline Socioeconomic variables & Statistics Denmark & Yes & Yes \\
\hline Quality of life at baseline and longitudinally & DD2 & Yes & Yes \\
\hline $\begin{array}{l}\text { Cardiovascular surrogate markers obtained at IDA } \\
\text { examinations }\end{array}$ & Study measurements & Yes & No \\
\hline Daily physical activity at IDA examinations & Study measurements & Yes & No \\
\hline
\end{tabular}

BMI, body mass index; DD2, Danish Centre for Strategic Research in Type 2 Diabetes; HbA1c, haemoglobin A1c; IDA, Individualised treatment of newly clinical diagnosed T2D in general practice study. 


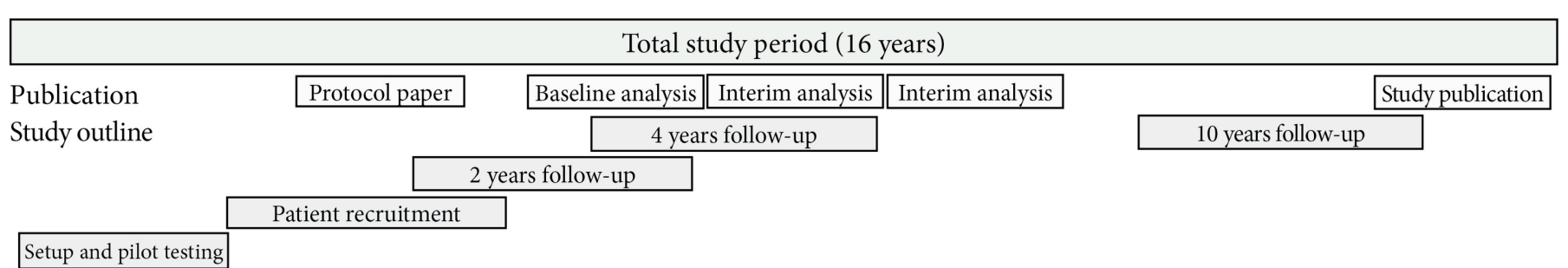

\section{OBJECTIVES}

The primary objective of the study is to assess the effect of individualised, multifactorial, interactive and supervised treatment in patients with T2D compared with treatment based on contemporary guidelines. The composite clinical outcome measure will encompass all-cause mortality, microvascular and macrovascular complications, cancer and hypoglycaemia. Secondary objectives are to assess effects on individual clinical outcomes, socioeconomic costs and quality of life.

\section{METHODS AND ANALYSIS \\ Setting and design}

The study is designed as a prospective controlled multicentre open-label study of a controlled intervention in the longitudinal DD2 cohort. Newly diagnosed patients with T2D are enrolled prospectively in the population-based DD2 cohort. At baseline, the DD2 project collects interview data and biobank blood and urine samples. ${ }^{34}{ }^{35}$ Following enrolment, each participant is followed over time using data in nationwide registries. ${ }^{36}$ The registries have documented high validity. ${ }^{37-39}$ The collected data in the study are summarised in table 1 . This study is one of several planned studies drawing on the cohort. ${ }^{40}$

The study setting will be community based. Patients in the intervention group will be recruited and treated by

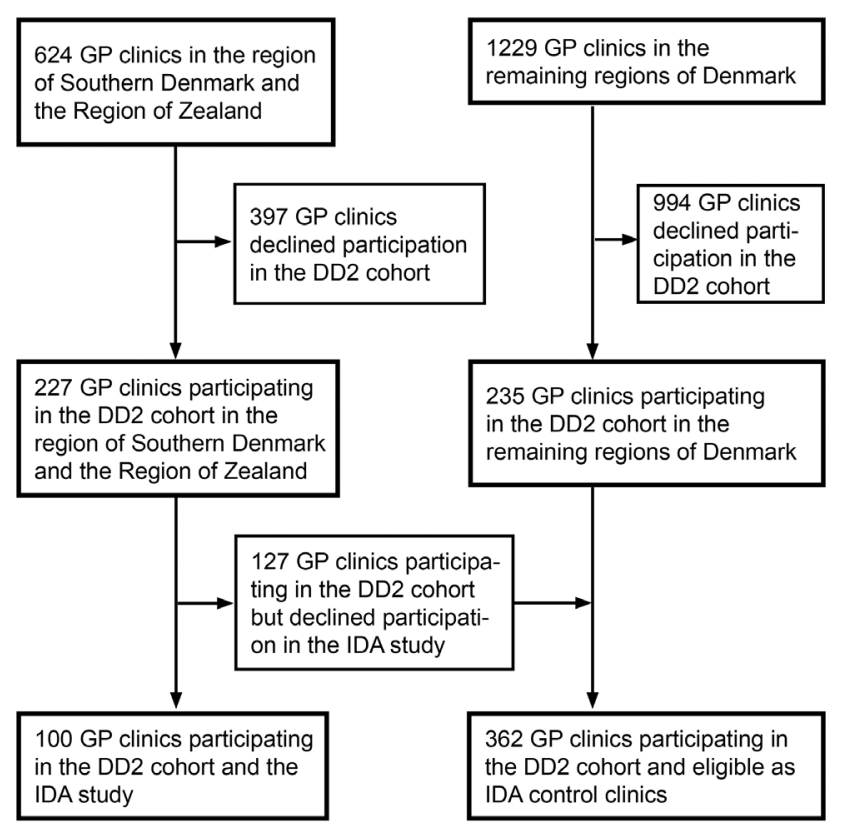

Figure 2 Recruitment flowchart at general practitioner (GP) level. DD2, Danish Centre for Strategic Research in Type 2 Diabetes; IDA, Individualised treatment of newly clinical diagnosed T2D in general practice study. 

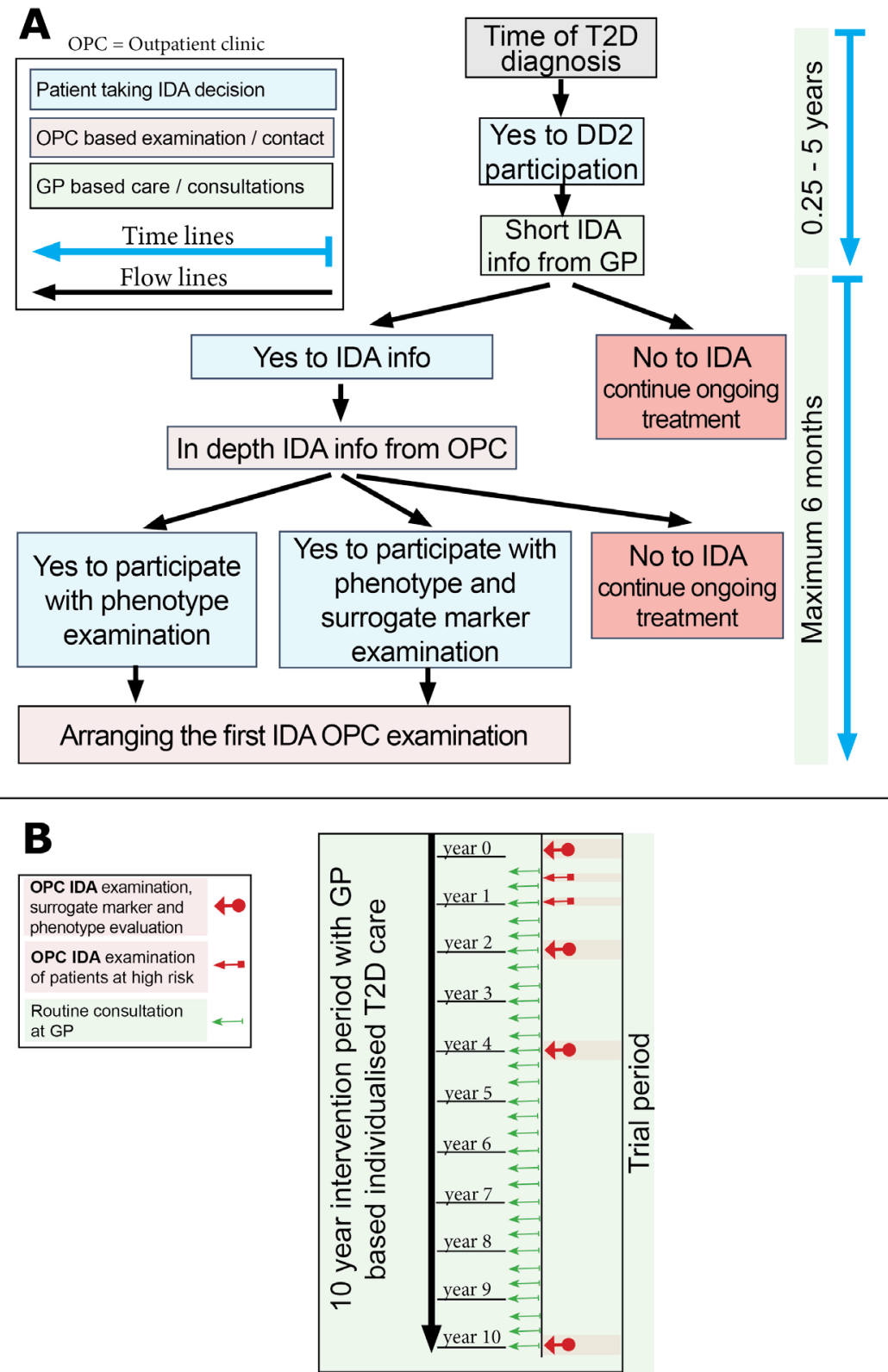

Figure 3 Recruitment (A) and general practitioner (GP) contacts in the intervention group (B). DD2, Danish Centre for Strategic Research in Type 2 Diabetes; IDA, Individualised treatment of newly clinical diagnosed T2D in general practice study; OPC, Outpatient clinic; T2D, type 2 diabetes.

study. Specialist counselling is also available by phone during the day.

\section{Patients and recruitment}

A flowchart of GPs participating in DD2 and IDA is provided in figure 2. Patients in the intervention group will be recruited from GPs in the region of southern Denmark and the region of Zealand participating in both the DD2 cohort and the IDA study. Patients in the control group will be recruited from GPs participating in the DD2 cohort but not in the IDA study. The selection process for patients in the two groups will be different and adjustment for differences in prognostic factors at baseline is therefore warranted as described in the statistical section.
Intervention patients

A flowchart of recruitment is provided in figure 3A. Participating IDA GPs will be responsible for the initial patient contact, including collection of brief general patient information. If a DD2 patient is interested in the study, the GP automatically will register the patient in the DD2 website, triggering contact by a study nurse who will give the patient detailed study information. This will occur either by phone or during an initial informational meeting. Collection of in-depth patient information, informed consent and additional screening will take place at central study hospitals prior to the baseline examination.

The following patient inclusion criteria will be used: 
- Member of the DD2 cohort. $^{42}$

- Patient at a GP participating in the IDA study.

- Not diagnosed with type 1 diabetes, defined as age $<30$ years at DD2 enrolment, fasting C-peptide $<300 \mathrm{pM}$ and GAD65-ab >20 IU/mL (see below).

- Life expectancy above 2 years.

- No participation in other clinical trials.

- Willing to provide written informed consent.

\section{Control patients}

Patients for the control group will be recruited from DD2 clinics throughout Denmark not participating in the IDA study (figure 2). Availability of valid biobanked samples for measuring GAD-ab, P-glucose and C-peptide will be required to join the control group. As the control group will be created within DD2, neither GP nor patient will be informed, ensuring that the control group is truly blinded.

\section{Patient examination in the intervention group}

Screening will take place at the following four central study sites: Odense University Hospital, Hospital of Southwest Denmark, Næstved Lægecenter and Holbæk Hospital. A written informed consent will be signed at the baseline visit prior to initiating examination of study participants.

Phenotype evaluation will be performed at the central sites at baseline and after 24 and 48 months. Medical history, medication use and measures used in the phenotype evaluation will be obtained from the patient. Fasting plasma glucose, GAD65-antibody and fasting C-peptide will be ascertained from the DD2 database. Repeat measures of cardiac impedance and unobserved automated blood pressure will be taken at the central sites at the following time points, determined by prior blood pressure values:

- Blood pressure (BP) $\leq 135 / 85 \mathrm{~mm} \mathrm{Hg:} \mathrm{impedance}$ measurement repeated after 24 months.

- 135/85 mm $\mathrm{Hg}<\mathrm{BP} \leq 145 / 95 \mathrm{~mm}$ Hg: impedance measurement repeated after 12 and 24 months.

- BP >145/95 mm Hg: impedance measurement repeated after 6,12 and 24 months.

The results of the phenotype evaluation for each specific patient will be assessed at Odense University Hospital and the patient-specific protocol recommendations sent to the patient's GP via the electronic health record.

Treatment and implementation of the phenotype evaluation will take place at the GP's office every third month or at the discretion of the GP (figure 3B). The GP will measure HbAlc, the lipid profile, the albumin-creatinine ratio, creatinine and BMI annually. The GP will report treatment goals and any reasons for protocol deviations annually.

\section{Daily physical activity}

To measure daily physical activity level, a Axivity AX3 accelerometer (Axivity, Newcastle, UK) will be taped on the thigh and on the lower back. The AX3 is an $11 \mathrm{~g}$ and $23 \times 32.5 \times 7.6 \mathrm{~mm}$ weatherproof accelerometer with a $512 \mathrm{MB}$ internal memory and clock. Accelerometers will be fixed directly on the skin using waterproof taping. Subjects will be instructed to wear the accelerometers at all times (including water activities and sleep) during a 10-day period and additional tape will be provided to patients at examination. The accelerometer on the back will be placed on the right side, above the upper point of the posterior iliac crest and next to the spine with its positive $\mathrm{x}$-axis pointing downward and its negative z-axis pointing forward. The accelerometer on the thigh will be placed on the medial front of the right thigh, midway between the hip and knee joints, with its positive $\mathrm{x}$-axis pointing downward and its negative $\mathrm{z}$-axis pointing forward. A sampling rate of $50 \mathrm{~Hz}$ will be used and data stored in the original cwa Axivity file format, but also converted into a binary gt $3 \mathrm{x}$ compatible file format using a custom-made add-on to OmGui Axivity software. Accelerometer wear time has recently been reported to be high. ${ }^{43}$ Patients will be closely instructed how to reattach the accelerometer in case it falls off.

\section{Cardiovascular surrogate markers}

Patients with clinically diagnosed T2D within 2 years of their baseline examination will be invited to participate in additional evaluation of the following cardiovascular surrogate markers:

1. 24-hour ambulatory blood pressure monitoring (24ABPM)

- 24ABPM will be implemented by means of brachial oscillometric measurements, using the Mobil-O-Graph system (IEM, Stolberg, Germany). The device will be set to measure BP every $15 \mathrm{~min}$ during the day (07:00-23:00) and every $30 \mathrm{~min}$ during the night (23:00-07:00). The patient will be instructed to record when $\mathrm{s} /$ he went to bed and got up.

2. Skin autofluorescence (SAF) to evaluate advanced glycosolated end-products

- SAF will be measured using the AGE Reader (DiagnOptics Technologies, Groningen, The Netherlands). Technical details of this non-invasive device have previously been described in detail. ${ }^{44}$

3. Low-dose non-contrast CT scan to detect coronary artery calcification

- The atherosclerotic plaque burden in the coronary, carotid, aortic and femoral arteries will be estimated by measuring calcium during a 64-slice CT scan (Discovery VCT; GE Healthcare, Milwaukee, Wisconsin, USA) conducted at Odense University Hospital. The scan will be performed with the following parameters: gantry rotation time 500 $\mathrm{ms}, 16 \times 2.5 \mathrm{~mm}$ collimation, $120 \mathrm{kV}$ tube voltage, $200 \mathrm{~mA}$ tube current and a prospectively ECG-triggered scan acquisition gating at $50 \%$ of the R-R interval. Scan data will be acquired during an inspiratory breath hold. The coronary artery calcium (CAC) Agatston score is computed by summing the CAC scores of all foci in the epicardial coronary system. 
4. Ultrasound of the carotid arteries to evaluate intimamedia thickness (IMT) and plaques

- IMT will be measured by B-mode ultrasound (Model IE33; Koninklijke Philips Electronics, Eindhoven, The Netherlands), using a linear array transducer (L11-3 with a frequency up to 11 $\mathrm{MHz}$ ), with acquisition of multi-insonation angles for subsequent analysis with automated edge detection software according to current guidelines. ${ }^{45}$

5. ECG for approximation of left ventricular hypertrophy (LVH)

- ECG will be measured digitally (EC Sense Lexor; Cardiolex, Solna, Sweden). The following measures of LVH will be calculated:

- Cornell voltage-duration product, defined as the sum of voltage of SV3 and RaVL multiplied by QRS duration (in women, $0.6 \mathrm{mV}$ is added to the voltage).

- Sokolow-Lyon voltage, defined as the sum of SV1 and R in V5 or V6, depending on which is larger.

6. Fundus photo to evaluate retinal vascular changes

- Retinal vascular changes will be assessed through retinal imaging. Two methods will be employed to assess diabetic retinopathy and vascular damage:

- Diabetic retinopathy will be graded using the Early Treatment of Diabetic Retinopathy Study classification. ${ }^{46}$ Grading is performed in seven standard fields. The assessed characteristics are graded in specific fields and/or multiple fields. The grading encompasses the following characteristics: microaneurysm, haemorrhages, hard exudates, soft exudates, intraretinal microvascular abnormalities, venous abnormalities, new vessels on disc or elsewhere, preretinal haemorrhage, vitreous haemorrhage, scars of prior photocoagulation and clinically significant macular oedema.

- The retinal arteriolar and venular calibre as described by Hubbard et al. ${ }^{47}$ All venules and arterioles in the area half to one disc diameter from the disc margin of the diameter are measured and an averaged measure is derived. The ratio of the venular and arteriolar diameters also is derived.

- Retinal photos will be taken after dilation of both eyes with one drop of $10 \%$ metaoxedrin and mydriacyl $5 \mathrm{mg} / \mathrm{mL}$. After $10 \mathrm{~min}$, this will be repeated. After a total of $20 \mathrm{~min}$, six pictures will be taken of each eye.

- All photographs will be assessed for retinopathy locally as part of the patients' regular screening. Trained ophthalmologists at the Department of Ophthalmology, Odense University Hospital, then will assess retinopathy with the methods described above.

Surrogate marker evaluation will take place at baseline and again at 2 and 4 years of follow-up.

\section{Study interventions}

Patients in the control group will not receive study-related interventions, but rather will be treated by their GP according to national guidelines. Patients in the intervention group will receive multifactorial individualised treatment as outlined below. The outline of treatment will be subject to revision during the study if substantial new clinical evidence emerges. The suggested individualised treatment in the intervention group is made available to the treating GP, but the actual treatment is chosen at the discretion of the GP together with the patient. The intervention is designed to mimic the actual real-life effect of specialist treatment suggestions. Patients who do not follow the proposed algorithms are therefore not discontinued.

\section{Antidiabetic treatment based on pathophysiological phenotypes}

Pathophysiological phenotyping will provide the basis for individually guided treatment. At inclusion, patients with 'other specific forms of diabetes' will be identified. The remaining patients with classical WHO-defined T2D will be characterised according to their insulin sensitivity (IS) and beta-cell function (BCF). Of note, the subphenotyping and treatment of patients with WHO-defined T2D is constructed for patients of Caucasian inheritance (very few Danish citizens are non-Caucasian) and cannot readily be extrapolated to other ethnicities.

Other specific types of diabetes are defined as follows:

- Maturity-onset diabetes of the young (MODY). Patients will be screened for 10 monogenic causes of diabetes.

- Late autoimmune diabetes of the adult (LADA). Defined as GAD65-ab $\geq 20 \mathrm{IU} / \mathrm{mL}$.

- Secondary diabetes. Defined as low BCF (HOMA2beta $<115.3 \%$ ) and a history of pancreatitis or pancreas resection.

- Steroid-associated diabetes, defined as use of oral glucocorticosteroids within 3 months of diabetes diagnosis. Genuine steroid-induced diabetes is present when it is known with certainty that diabetes was not present in the 3 months prior to steroid initiation. Patients with known prior T2D or with uncertainty about the timing of diabetes onset and steroid use will be allocated to one of the additional phenotypes described below.

Genuine WHO-defined T2D:

- Insulinopaenic T2D. Defined as low BCF (HOMA2beta $<115.3 \%$ ) and high IS (HOMA2-S $\geq 63.5 \%$ ).

- Classical T2D. Defined as low BCF (HOMA2beta $<115.3 \%$ ) and low IS (HOMA2-S $<63.5 \%$ ).

- Hyperinsulinaemic T2D. Defined as high BCF (HOMA2-beta $2115.3 \%$ ) and low IS (HOMA2-S $<63.5 \%$ ).

The classification is hierarchical. The phenotypes have been described previously. ${ }^{25} \mathrm{BCF}$ and IS will be assessed using the HOMA2 model, calculated based on fasting C-peptide and fasting plasma glucose. HOMA2-beta is an estimate of the BCF and HOMA2-S is an estimate of the 
IS. In a healthy population without diabetes or impaired glucose tolerance, median HOMA2-beta was found to be $115.3 \%$ and median HOMA2-S was $63.5 \%$. In the study population, values of HOMA2-beta or HOMA2-S above these medians will be defined as 'high', while values below the median will be defined as 'low'.

Proposed treatment strategies in the study according to diabetes phenotypes are as follows. The treatment proposed for each phenotype is additive, starting with A. $\mathrm{B}$ is added if the treatment goal is not reached, and so on.

1. MODY. (1) Types 1 and 3 MODY should be treated with glimepiride or repaglinid. (2) Type 2 MODY should be treated with diet. Secondarily, basal insulin can be used. (3) Type 5 MODY should be treated with basal insulin. (4) Rare types of MODY should be treated individually according to specialist assessment.

2. LADA.

- In patients who have IS and BCF equivalent to insulinopaenic and classic T2D:

- (A) Metformin if BMI $>25 \mathrm{~kg} / \mathrm{m}^{2}$. (B) Basal insulin and meal-time insulin.

- In patients who have IS and BCF equivalent to hyperinsulinaemic T2D:

- Treatment equivalent to hyperinsulinaemic T2D (see below), with additional information on risk of rapid BCF deterioration and possible absolute need for insulin therapy.

3. Secondary diabetes. Basal and meal-time insulin.

4. Steroid-associated diabetes. (A) Meal-time insulin. (B) Metformin. (C) Basal insulin if fasting blood glucose is above 7.0.

5. Insulinopaenic T2D. (A) Metformin. (B) Basal insulin. (C) Meal-time insulin.

6. Classical T2D.

- In patients without CVD: (A) Metformin. (B) GLP-1 analogue*. (C) Basal insulin. (D) Meal-time insulin.

- In patients with former CVD: (A) Metformin. (B) SGLT-2 inhibitor. (C) GLP-1 analogue*. (D) Basal insulin. (E) Meal-time insulin.

7. Hyperinsulinaemic T2D. In patients with BMI $>35 \mathrm{~kg} /$ $\mathrm{m}^{2}$, gastric bypass should be considered according to current national criteria and patient preference. Pharmacological treatment:

- In patients without CVD: (A) Metformin. (B) GLP1 analogue*. (C) Pioglitazone. (D) Basal insulin. (E) Meal-time insulin.

- In patients with former CVD: (A) Metformin. (B) SGLT-2 inhibitor. (C) GLP-1 analogue*. (D) Pioglitazone. (E) Basal insulin. (F) Meal-time insulin.

Pioglitazone is not recommended for patients with heart failure, prior bladder cancer or known osteoporosis. If marked oedema develops, discontinuation of pioglitazone must be considered. Women should be informed about the increased risk of fractures with pioglitazone, alongside the reduced cardiovascular risk.
*DDP-4 inhibitors can be used if the patient does not want a GLP-1 analogue. It is recommended that a DDP-4 inhibitor be discontinued if insulin is initiated.

In patients of Asian inheritance with WHO-defined T2D, incretin-based treatment can be considered as first-line treatment. ${ }^{48}$ Type of drug within drug classes, dosing and titration will be chosen at the discretion of the treating physician. Suggestive algorithms will be available to the physicians.

Treatment of hyperglycaemia will proceed according to the following individual goals:

- Optimal control of HbAlc $<48 \mathrm{mmol} / \mathrm{mol}$;

- Acceptable control of HbAlc $<58 \mathrm{mmol} / \mathrm{mol}$; or

- Free of symptoms, with best possible HbAlc achieved within this constraint.

All treatment algorithms will be applied according to these predetermined goals. GPs will be free to choose and reassess the goal applicable to an individual patient. In patients with neuropathy or pre-existing cardiovascular disease, careful goal assessment is needed. If a patient has a severe hypoglycaemic event, has repeated measures of blood glucose below $4.0 \mathrm{mmol} / \mathrm{L}$ or is therapy resistant, the goal should be reassessed. For an in-depth discussion of the motivation for the glucose-lowering algorithm, we refer to the online supplementary material.

\section{Antihypertensive treatment}

Treatment of hypertension will be guided by measurements of thoracic impedance, which provide estimates of vascular resistance, intravascular volume and cardiac inotropy. These measurements will be used to guide the pharmacological treatment of arterial hypertension. Principles of drug class choice will be as follows:

- When hypertension or microalbuminuria are present, patients should be treated with an ACE inhibitor (or a angiotensin-2 antagonist), regardless of the result of the impedance measurement.

- High vascular resistance, as the only abnormal impedance measure, should be treated with a calcium-channel blocker (CCB) (dihydropyridins) in addition to an ACE inhibitor.

- High intravascular volume, as the only abnormal impedance measure, should be treated with a thiazide in addition to current antihypertensive treatment. If the patient already is receiving a thiazide in maximum dose, an aldosterone receptor antagonist should be added.

- In cases of high vascular resistance and high intravascular volume (more than double the vascular resistance in relative terms), diuretics (thiazide or secondarily aldosterone receptor antagonist) should be increased, comparable to the maximum dose of one new drug. As a next step, a CCB should be added. Finally, an aldosterone receptor antagonist should be increased to its maximum dose.

- In cases of high vascular resistance and high intravascular volume (but less than double the vascular resistance in relative terms), an ACE inhibitor or 
CCB should be added, depending on initial treatment. As a second measure, diuretics (thiazide or, less often, an aldosterone receptor antagonist) should be increased comparable to the maximum dose of one new drug. As a third measure, CCB should be titrated to its maximum dose. Finally, an aldosterone receptor antagonist can be increased to its maximum dose.

- High inotropy is addressed only when the patient receives an ACE inhibitor, thiazide and $\mathrm{CCB}$ and the impedance measurement is made while the patient is receiving this treatment. Other abnormalities need to be addressed first. Carvedilol up to $50 \mathrm{mg}$ is recommended.

The maximum dose of bendroflumethiazide is considered to be $5.0 \mathrm{mg}$, that for hydrochlorthiazide is $50 \mathrm{mg}$ and that for spironolactone is $50 \mathrm{mg}$. In cases in which the estimated glomerular filtration rate (eGFR) is below $30 \mathrm{~mL} / \mathrm{min} / 1.73 \mathrm{~m}^{2}$, high ceiling diuretics are substituted for thiazides. Non-hypertensive indications for antihypertensive medication overrule this algorithm.

Impedance measurements also are used to downgrade antihypertensive treatment, in the following situations:

- When inotropy is decreased, beta-blockers are terminated or the dose reduced.

- When hypovolaemia is present, diuretics are terminated or the dose reduced.

- When inotropy is decreased, vascular resistance is normal and blood pressure is regulated, CCBs can be reduced or terminated.

For patients with prior cardiovascular disease, chronic kidney disease (eGFR $<60 \mathrm{~mL} / \mathrm{min} / 1.73 \mathrm{~m}^{2}$ ) or albuminuria, the goal of antihypertensive treatment will be to achieve a blood pressure $<130 / 80 \mathrm{~mm} \mathrm{Hg}$ on three drugs, measured as home blood pressure or with an automated blood pressure device. For other patients, the goal is blood pressure $<135 / 80 \mathrm{~mm} \mathrm{Hg}$ on three drugs. In cases in which control of blood pressure is not achieved with three antihypertensive drugs, the patient will be referred to a specialist clinic. Blood pressure below $120 / 70 \mathrm{~mm} \mathrm{Hg}$ should be avoided, if necessary by means of down-titration, unless other considerations are present.

Hypertension at study inclusion will be defined by presence of antihypertensive treatment or a blood pressure measurement above or equal to $135 / 80 \mathrm{~mm} \mathrm{Hg}$ in the office under standardised conditions using an automated blood pressure device (Mobilograf).

\section{Treatment of dyslipidaemia}

Treatment with atorvastatin or simvastatin $40 \mathrm{mg}$ will be recommended for all patients, regardless of the level of low-density lipoprotein cholesterol (LDL-C). If the treatment goal is not met, atorvastatin $80 \mathrm{mg}$ or rosuvastatin $40 \mathrm{mg}$ will be recommended. Combining lipid-lowering drugs will not be recommended. The treatment goal will be LDL cholesterol $\leq 2.5 \mathrm{mM}$ and LDL cholesterol $\leq 2.0 \mathrm{mM}$ in patients with established CVD.
Termination of inefficient medication

The effect of a specific antidiabetic, antihypertensive or cholesterol-lowering treatment will be measured. The following efficacy requirements after titration to the full tolerable dose will need to be met:

- Decline in HbA1c exceeding $0.5 \%$ within 3 months.

- Decline in systolic blood pressure exceeding $5 \mathrm{~mm} \mathrm{Hg}$ within 1 month.

- Decline in LDL cholesterol exceeding $0.5 \mathrm{mM}$ within 1 month.

If the target is not met, the specific treatment should be terminated and replaced by another drug according to the algorithm. The missing effect on blood pressure should be validated by home blood pressure measurements according to national guidelines. For antihypertensive medication, another drug within the same class can be tried.

\section{Lifestyle interventions}

Supportive individualised M-health initiatives and faceto-face consultations for promoting changes in lifestyle will be used in this study. The aim is to empower patients to achieve sustainable reductions in carbohydrate intake and increase the daily physical activity level.

In the current study, we will integrate dietician-supported self-management by using a commercially Internet platform (Liva) to facilitate interactive communication between dieticians and users, as well as by peerto-peer support. The platform will be further developed to support individualised education, goal setting and evaluation of diet and exercise behaviour as described below. In addition, interactive communication between patient and a personal healthcare professional will allow for asynchronous contact when needed, in the form of video, text or spoken messages. The advanced interactive platforms will be supported further by an individualised number of face-to-face consultations between the patient and the personal healthcare professional, with whom the patient will be acquainted from the interactive platform.

The current study aims to empower patients to decrease the amount of carbohydrates in their diet ( $40 \%$ fat, $40 \%$ carbohydrates and 20\% proteins), while keeping energy intake unchanged or slightly decreased in cases in which the patient seeks weight reduction. We will support individualised changes towards a sustainable increased number of low-carbohydrate meals each week. To support these changes, we have designed an Internet and smartphone platform (www.dd2mad.dk) that easily allows individuals to plan, purchase groceries and cook low-carbohydrate meals for all daily meals, including snacks. A dietician developed the recipes, and the macronutritional composition of each recipe has been calculated using 'DANKOST' software (Dankost Aps, Copenhagen, Denmark). The platform is updated monthly with new recipes and will be developed further using a user-driven iterative process. We will implement this platform in the modified Liva. 
We aim to empower patients to make a sustainable increase in physical activity level by implementing interval walking. To enable the patients to engage in and maintain correct individualised interval walking, we have developed a smartphone application (InterWalk). The application is designed to individually guide duration and intensity of the training in real time based on a small test at the first use of the application. During use, the app automatically monitors exercise intensity, training duration and walking distance. Following each training session, the app sends the data to a central server. ${ }^{49}$ The training data will be available for the patient and the healthcare professionals making it possible to provide evidence-based training feedback to patients, using physical fitness data, ${ }^{50}$ training duration and compliance from the app. ${ }^{49}$ The InterWalk app will be implemented in the Liva platform. For a discussion of the motivation of the lifestyle intervention, see the online supplementary material.

\section{Outcomes}

The study's primary outcome measure is time to a composite outcome of all-cause mortality, non-fatal myocardial infarction, coronary revascularisation, cardiac arrest with resuscitation, hospitalisation for heart failure, non-fatal stroke, development or progression of nephropathy or retinopathy (see below), severe hypoglycaemia leading to hospitalisation and development of any cancer (except basocellular carcinoma). Development or progression of nephropathy is defined as renal failure (defined by the need for chronic dialysis), development of macroalbuminuria or doubling of baseline s-creatinine to a level above $200 \mu \mathrm{M}$. Development of retinopathy is defined as proliferative retinopathy or macular oedema that requires laser therapy, injection of vascular endothelial growth factor inhibitors or vitrectomy, or diabetes-related blindness (Snellen visual acuity below 0.1 ). Information on deaths will be obtained from the Civil Registration System. Individual diagnoses, operations and procedure codes will be obtained from the Danish National Patient Registry (see table 2). S-creatinine values and albumin-creatinine ratios will be obtained through the Danish Diabetes Database for Adults.

Secondary outcome measures are all-cause mortality, socioeconomic costs and quality of life. Quality of life will be assessed with SF-12 and Q-5 questionnaires at study inclusion and after 2 and 4 years.

Tertiary outcome measures are the individual endpoints in the composite endpoint. Other endpoints are

- Time to any macrovascular endpoint (as defined in the primary endpoint).

- Time to any microvascular endpoint (as defined in the primary endpoint).

- Time to fatal acute myocardial infarction.

Table 2 Endpoint definitions 10th International Classification of Diseases (ICD-10) codes for diagnoses and operation codes were obtained from the Danish National Patient Registry, listed below)

\begin{tabular}{ll}
\hline Non-fatal myocardial infarction & I21-23, T822A, T823 (without death within 30 days) \\
\hline Coronary revascularisation & KFNG, KFNA, KFNB, KFNC, KFND, KFNE, KFNF, KFNH, KFNW, KFLF \\
Cardiac arrest with resuscitation & 146 \\
Hospitalisation for heart failure & $150,111.0$, I13.0+2 (only as a diagnosis) \\
Non-fatal stroke (including cerebral & I61, I63, I64, KAAL10, KAAL11 (without death within 30 days)
\end{tabular}
haemorrhage)

Development of nephropathy

BJFD2 (chronic dialysis) or

urine albumin-creatinine ratio $>300 \mathrm{~g} / \mathrm{mg}$ or doubling of creatinine (if creatinine $\geq 200 \mu \mathrm{M}$ )

Development of retinopathy

KCKC10, KCKC15 (laser therapy) or

DH360K and KCKD05B

KCKD65 (vitrectomy) or

H540+1+4 (blindness)

Severe hypoglycaemia leading to
hospitalisation
Cancer (except basocellular carcinoma)
Amputation of the lower limbs:
Revascularisation procedures and
peripheral thrombosis (not cardiovascular
or cerebrovascular disease)

E15, E160-2, T383A

C00-99 (except when ZM809xx are added) KNEQ, KNFQ, KNGQ, KNHQ

I74, N280, K550-1, K558-9, H340-2

or cerebrovascular disease)

$\mathrm{KPBE}+\mathrm{F}+\mathrm{H}+\mathrm{N}+\mathrm{p}+\mathrm{Q}, \mathrm{KPBW}, \mathrm{KPGH} 10, \mathrm{KPGE}+\mathrm{F}+\mathrm{H}+\mathrm{N}+\mathrm{p}+\mathrm{Q}, \mathrm{KPGW} 99, \mathrm{KPGW} 20$, $\mathrm{KPEE}+\mathrm{F}+\mathrm{H}+\mathrm{N}+\mathrm{p}+\mathrm{Q}+\mathrm{W}, \mathrm{KPFE}+\mathrm{H}+\mathrm{n}+\mathrm{P}+\mathrm{Q}+\mathrm{W}, \mathrm{KPGH} 20+21+22+23+30+31+40+99$, $\mathrm{KPDU} 74+82+83+84, \mathrm{KPEU} 74+82+83+84, \mathrm{KPFU} 74+82+83+84, \mathrm{KPAE}+\mathrm{F}+\mathrm{H}+\mathrm{N}+\mathrm{p}+\mathrm{Q}$, KPAW99, KPAU74, KPCE+F+H+N+p+Q, KPCW99, KPCW20, KPCU74+82+83+84, KPGU74+83+84+99, KPGW, KPWG

Fatal acute myocardial infarction I21-23, T822A, T823, R96-99 with death within 30 days

Fatal stroke 161, I63, 164 with death within 30 days 
- Time to fatal stroke.

- Time to lower-limb amputation

- Time to other revascularisation procedures and peripheral thrombosis (not cardiac or cerebrovascular events).

- Overall hospitalisations per 1000 patient-years.

- Treatment adherence, defined as reimbursement of prescriptions, compared with intended treatment dose: total yearly reimbursed doses versus intended yearly doses.

- Time trends of HbAlc, blood pressure and LDL cholesterol.

- Individual time trends of daily physical activity in intervention patients.

- Proportions of patients in the interventions group reaching their goal of HbAlc and blood pressure.

Cause of death will be obtained from the Danish Cause of Death Register. Reimbursement of prescriptions will be ascertained through the Danish National Prescription Registry. Intended treatment dose will be obtained through the electronic medicine chart 'FMK'.

\section{Power calculations}

The power calculation was performed using Lakatos normal approximation for a log-rank test of two survival curves. The estimated sample size of 1123 patients will have $80 \%$ power to detect a reduction of $20 \%$ of the incidence rate of the primary endpoint, with a type 1 error of $5 \%$, during 10 years of follow-up. A composite yearly event rate of $5 \%$ is estimated, based on estimated incidence rates of $2.5 \%$ per year of macrovascular and microvascular complications, $1.5 \%$ per year for cancer and approximately $1 \%$ for overall mortality. The hypoglycaemic event rate is expected to be less than $0.4 \%$. Due to the database approach, loss to follow-up should be minimal.

\section{Statistical analysis}

The advantage of our pragmatic study approach is that our results will reflect effectiveness, harms and costs of individualised treatment in daily practice in primary care, improving generalisability compared with single-exposure RCTs typically conducted among heavily selected patient and clinic populations. On the other hand, GP practices and their patients are self-selected to participating in the IDA intervention in our study and will be non-blinded to receiving this treatment. The main methodological challenge for our proposed study will therefore be to address possible confounding caused by imbalance of prognostic factors in participants versus controls. We will use appropriate statistical methods for dealing with confounding, including regression analyses and propensity score matching.

\section{Confounders}

Covariates expected to be confounders will be selected according to available evidence and knowledge, and will include the following:
- General variables: age, gender, diabetes onset, DD2 enrolment year, time from DD2 enrolment to IDA study entry, GP and place of residence (municipality).

- Lifestyle variables: smoking, alcohol consumption and physical exercise (self-reported).

- Comorbidity: each of the individual strata of the Charlson Comorbidity Index, except diabetes, hospitalisation for hypoglycaemia, chronic dialysis, laser treatment of retinopathy/maculopathy, vitrectomy, chronic heart disease, angina pectoris, any revascularisation procedure, lower-extremity amputation, atrial fibrillation and history of psychiatric disease (eight covariates defined by 10th International Classification of Diseases (ICD-10): DF1 to DF8).

- Socioeconomic variables: education, employment, income and social support.

- Clinical variables: blood pressure, BMI, waist circumference, LDL-C, high-density lipoprotein cholesterol (HDL-C), triglyceride level, creatinine level, urine albumin-creatinine ratio and diabetes phenotype.

- Medication use: aspirin, statins, anticoagulating drugs, thiazides, ACE inhibitors or angiotensin-2 antagonists, calcium channel antagonists, beta-blockers, potassium-sparing diuretics, metformin, sulfonylurea, DD4 inhibitors, GLP-1 analogues, SGLT-2 inhibitors, insulin, oral corticosteroids and number of redeemed drugs (including the above drugs).

Ascertainment of confounder variables will be through the DD2 cohort and registries. Comorbidity is defined as all diagnoses registered from 1977 until enrolment. Socioeconomic variables are defined as the values recorded in the enrolment year. Medication use is defined as redeemed prescriptions 1 year prior to enrolment. Clinical variables are defined by the value measured closest to enrolment, not more than 1 year prior to enrolment and 1 month after enrolment.

\section{Cox regression analysis}

Follow-up will extend for 10 years from the date of IDA intervention start until first of any of the individual composite outcome events, emigration out of Denmark or end of study (1 January 2028), whichever comes first. For patients in the control group, the entry date will be the date of DD2 enrolment or date of the overall IDA study initiation (1 January 2015), whichever comes last. We will construct survival curves for intervention and control patients and compute cumulative incidence rates. We then compute incidence rate ratios with $95 \%$ CIs for intervention patients compared with controls using Cox regression analysis and controlling for confounders described above. Stratified analyses will be performed by gender, age below/above 60 years, eGFR below/above 60 , former CVD and diabetes phenotype with test for interaction.

\section{Propensity score analysis}

In a second analysis, we will use propensity score matching. For this analysis, we will compute the probability of each 
DD2 cohort non-IDA-intervention patient being included in the IDA intervention arm in a logistic regression analysis, conditioned on the patient's covariate profile. Next, we will match each intervention patient to a DD2 control patient with the closest propensity score in a 1:3 fashion and eliminate the remaining controls. The matching will be performed in a random sequential order. After determining that the covariates are balanced between the two treatment groups (see online supplementary material), we will conduct a matched Cox regression analysis without further adjustment. If any covariate is not balanced, a model with adjustment for non-balanced covariates will be made. The assumption of proportional hazards in the Cox models will be assessed graphically.

\section{ETHICS AND DISSEMINATION}

The study will use well-known pharmalogical agents and bariatric interventions. Thus, the safety of the patients is considered high. Patients in the intervention group will provide written informed consent before participation. The study will be conducted in compliance with the principles set forth in the Declaration of Helsinki and the Good Clinical Practice Guidelines. The study has been approved by the Regional Committee on Medical Health Ethics (Region of Southern Denmark S-20120186), the Danish Data Protection Agency and the Danish Health and Medicines Authority (journal no. 2012120204).

All subjects will be identified by an unambiguous subject code that can be linked to the civil registration number. The subject code will be used as a pseudoanonymisation code throughout the study. Handwritten source data (CRFs) or hard-copy source data will be securely safeguarded against unauthorised access and kept under lock, with access only by authorised persons. Electronically reported source data will conform to good clinical practice standards by using the RedCap data collection system and the DD2-established data collection system. Both systems have a high level of security and use data verification and detailed logging during reporting.

Most study data will be stored in OPEN, a custom-designed study database secured against unauthorised access. OPEN is a research service provided by the University of Southern Denmark that enables researchers to store research data in accordance with national legislation and requirements for data logging, password security and backup.

Study results will be made public via articles in national and international peer-reviewed journals, which will be accessible online https://dd2.nu). Positive, negative and inconclusive results will be published according to the Vancouver Principles. The results will be disseminated through www.clinicaltrials.com and the Danish Diabetes Association.

\section{PERSPECTIVES}

IDA is one of the first studies to formalise a specific implementation of individualised medicine in treating T2D. The ultimate goal is to improve quality of life and reduce complications in patients with $\mathrm{T} 2 \mathrm{D}$-in a manner requiring less medication and fewer resources over a 10-year period.

Contributors The study concept was developed by HB-N and JEH. HB-N, JEH, MHO, JVS, JSN, TBO, RWT and SGF designed the study. Principal manager of the DD2 study is JSN. JVS drafted the article and JSN, RWT, HB-N and MHO revised the draft. All authors participated in the critical revision of the intellectual content of the report.

Funding The study was supported by the Danish Agency for Science (grant nos. 09-067009 and 09-075724), the Region of Southern Denmark, the Region of Zealand, the Augustinus Foundation, the Herta Christensen Foundation, the Novo Nordisk Foundation and the University of Southern Denmark. The Biobank was supported by an unrestricted donation from Novo Nordisk A/S. Project partners are listed on the project website (https://DD2.nu).

Competing interests JEH is a member of the MSD and Boehringer National Advisory Board. HB-N has received personal lecturer's fees from Novo Nordisk, outside the submitted work. MHO has received a clinical research grant from the Novo Nordisk Foundation. The Department of Clinical Epidemiology, Aarhus University Hospital, receives funding for other studies from companies in the form of research grants to (and administered by) Aarhus University. None of those studies have any relation to the present study.

Ethics approval Regional Committee on Medical Health Ethics.

Provenance and peer review Not commissioned; externally peer reviewed.

Open Access This is an Open Access article distributed in accordance with the Creative Commons Attribution Non Commercial (CC BY-NC 4.0) license, which permits others to distribute, remix, adapt, build upon this work non-commercially, and license their derivative works on different terms, provided the original work is properly cited and the use is non-commercial. See: http://creativecommons.org/ licenses/by-nc/4.0/

(c) Article author(s) (or their employer(s) unless otherwise stated in the text of the article) 2017. All rights reserved. No commercial use is permitted unless otherwise expressly granted.

\section{REFERENCES}

1. Gæde P, Oellgaard J, Carstensen B, et al. Years of life gained by multifactorial intervention in patients with type 2 diabetes mellitus and microalbuminuria: 21 years follow-up on the Steno-2 randomised trial. Diabetologia 2016;59:1-10.

2. Gregg EW, Sattar N, Ali MK. The changing face of diabetes complications. Lancet Diabetes Endocrinol 2016;4:537-47.

3. Færch K, Carstensen B, Almdal TP, et al. Improved survival among patients with complicated type 2 diabetes in Denmark: a prospective study (2002-2010). J Clin Endocrinol Metab 2014;99:E642-6.

4. Coutinho M, Gerstein HC, Wang Y, et al. The relationship between glucose and incident cardiovascular events. A metaregression analysis of published data from 20 studies of 95,783 individuals followed for 12.4 years. Diabetes Care 1999;22:233-40.

5. Currie CJ, Peters JR, Tynan A, et al. Survival as a function of $\mathrm{HbA}(1 \mathrm{c})$ in people with type 2 diabetes: a retrospective cohort study. Lancet 2010;375:481-9.

6. Gerstein HC, Miller ME, Byington RP, et al. Effects of intensive glucose lowering in type 2 diabetes. $N$ Engl J Med 2008;358:2545-59.

7. Patel A, MacMahon S, Chalmers J, et al. Intensive blood glucose control and vascular outcomes in patients with type 2 diabetes. $N$ Engl J Med 2008;358:2560-72.

8. Duckworth W, Abraira C, Moritz T, et al. Glucose control and vascular complications in veterans with type 2 diabetes. $N$ Engl J Med 2009;360:129-39.

9. Boussageon R, Bejan-Angoulvant T, Saadatian-Elahi M, et al. Effect of intensive glucose lowering treatment on all cause mortality, cardiovascular death, and microvascular events in type 2 diabetes: meta-analysis of randomised controlled trials. BMJ 2011;343:d4169.

10. Seidu S, Achana FA, Gray LJ, et al. Effects of glucose-lowering and multifactorial interventions on cardiovascular and mortality outcomes: a meta-analysis of randomized control trials. Diabet Med 2016;33:280-9.

11. Anon. Effect of intensive blood-glucose control with metformin on complications in overweight patients with type 2 diabetes (UKPDS 
34). UK Prospective Diabetes Study (UKPDS) Group. Lancet 1998;352:854-65

12. Anon. Intensive blood-glucose control with sulphonylureas or insulin compared with conventional treatment and risk of complications in patients with type 2 diabetes (UKPDS 33). UK Prospective Diabetes Study (UKPDS) Group. Lancet 1998;352:837-53.

13. Holman RR, Paul SK, Bethel MA, et al. 10-year follow-up of intensive glucose control in type 2 diabetes. N Engl J Med 2008;359:1577-89.

14. Svensson E, Baggesen LM, Johnsen SP, et al. Early glycemic control and magnitude of $\mathrm{HbA} 1 \mathrm{c}$ reduction predict cardiovascular events and mortality: population-based cohort study of 24,752 metformin initiators. Diabetes Care 2017;40:800-7.

15. Gerstein HC, Miller ME, Genuth S, et al. Long-term effects of intensive glucose lowering on cardiovascular outcomes. $N$ Engl $J$ Med 2011;364:818-28.

16. Zoungas S, Patel A, Chalmers J, et al. Severe hypoglycemia and risks of vascular events and death. N Engl J Med 2010;363:1410-8.

17. American Diabetes Association. Standards of medical care in diabetes 2017. Diabetes Care 2017;40(Suppl 1):S4-5.

18. Noto H, Tsujimoto T, Sasazuki T, et al. Significantly increased risk of cancer in patients with diabetes mellitus: a systematic review and meta-analysis. Endocr Pract 2011;17:616-28.

19. Shikata K, Ninomiya T, Kiyohara Y. Diabetes mellitus and cancer risk: review of the epidemiological evidence. Cancer Sci 2013;104:9-14.

20. Campbell PT, Newton CC, Patel AV, et al. Diabetes and causespecific mortality in a prospective cohort of one million U.S. adults. Diabetes Care 2012;35:1835-44.

21. Wu L, Zhu J, Prokop LJ, et al. Pharmacologic therapy of diabetes and overall cancer risk and mortality: a meta-analysis of 265 studies. Sci Rep 2015;5:10147.

22. Expert Committee on the Diagnosis and Classification of Diabetes Mellitus. Report of the expert committee on the diagnosis and classification of diabetes mellitus. Diabetes Care 2003;26(Suppl 1):S5-20.

23. Tuomi T, Santoro N, Caprio S, et al. The many faces of diabetes: a disease with increasing heterogeneity. Lancet 2014;383:1084-94.

24. American Diabetes Association. Diagnosis and classification of diabetes mellitus. Diabetes Care 2014;37(Suppl 1):S81-90.

25. Stidsen JV, Thomsen RW, Nielsen JS, et al. Pathophysiological phenotypes of clinically diagnosed type 2 diabetes. abstracts of the 74th Scientific Sessions of the American Diabetes Association, June 13-17, 2014, San Francisco, California. Diabetes 2014;63(Suppl 1):A354-5

26. Kodama K, Tojjar D, Yamada S, et al. Ethnic differences in the relationship between insulin sensitivity and insulin response: a systematic review and meta-analysis. Diabetes Care 2013;36:1789-96.

27. Yabe D, Seino Y. Type 2 diabetes via $\beta$-cell dysfunction in east Asian people. Lancet Diabetes Endocrinol 2016;4:2-3.

28. Stamler J, Vaccaro O, Neaton JD, et al. Diabetes, other risk factors, and 12-yr cardiovascular mortality for men screened in the Multiple Risk Factor Intervention Trial. Diabetes Care 1993;16:434-44.

29. Brunström M, Carlberg B. Effect of antihypertensive treatment at different blood pressure levels in patients with diabetes mellitus: systematic review and meta-analyses. BMJ 2016;352:i717.

30. Mancia G, Fagard R, Narkiewicz K, et al. 2013 ESH/ESC practice guidelines for the management of arterial hypertension. Blood Press 2014;23:3-16.

31. Ferrario CM, Flack JM, Strobeck JE, et al. Individualizing hypertension treatment with impedance cardiography: a metaanalysis of published trials. Ther Adv Cardiovasc Dis 2010;4:5-16.

32. Fadl Elmula FE, Rebora P, Talvik A, et al. A randomized and controlled study of noninvasive hemodynamic monitoring as a guide to drug treatment of uncontrolled hypertensive patients. J Hypertens 2015;33:2534-45.

33. International Diabetes Federation Guideline Development Group. Global guideline for type 2 diabetes. Diabetes Res Clin Pract 2014;104:1-52.
34. Steffensen C, Thomsen RW, Vaag A, et al. The Danish Centre for Strategic Research in Type 2 Diabetes (DD2) Project: rationale and planned nationwide studies of genetic predictors, physical exercise, and individualized pharmacological treatment. Clin Epidemiol 2012;4(Suppl 1):7-13.

35. Nielsen JS, Thomsen RW, Steffensen C, et al. The Danish Centre for Strategic Research in Type 2 Diabetes (DD2) study: implementation of a nationwide patient enrollment system. Clin Epidemiol 2012;4(Suppl 1):27-36.

36. Thomsen RW, Friborg S, Nielsen JS, et al. The Danish Centre for Strategic Research in Type 2 Diabetes (DD2): organization of diabetes care in Denmark and supplementary data sources for data collection among DD2 study participants. Clin Epidemiol 2012;4(Suppl 1):15-19.

37. Schmidt M, Schmidt SA, Sandegaard JL, et al. The Danish National Patient Registry: a review of content, data quality, and research potential. Clin Epidemiol 2015;7:449-90.

38. Thomsen RW, Pedersen L, Møller N, et al. Incretin-based therapy and risk of acute pancreatitis: a nationwide population-based casecontrol study. Diabetes Care 2015;38:1089-98.

39. Pottegård A, Schmidt SAJ, Wallach-Kildemoes $\mathrm{H}$, et al. Data resource profile: the Danish National Prescription Registry. Int $J$ Epidemiol 2017;46:dyw213.

40. Beck-Nielsen H, Solomon TP, Lauridsen J, et al. The Danish Centre for Strategic Research in Type 2 Diabetes (DD2) study: expected outcome from the DD2 project and two intervention studies. Clin Epidemiol 2012;4(Suppl 1):21-6.

41. Christensen H, Nielsen JS, Sørensen KM, et al. New National Biobank of The Danish Center for Strategic Research on Type 2 Diabetes (DD2). Clin Epidemiol 2012;4:37-42.

42. Thomsen RW, Nielsen JS, Ulrichsen SP, et al. The Danish Centre for Strategic Research in Type 2 Diabetes (DD2) study: collection of baseline data from the first 580 patients. Clin Epidemiol 2012;4:43-8.

43. Schneller MB, Bentsen P, Nielsen G, et al. Measuring children's physical activity: compliance using skin-taped accelerometers. Med Sci Sports Exerc 2017;49:1261-9.

44. Meerwaldt R, Graaff R, Oomen PHN, et al. Simple non-invasive assessment of advanced glycation endproduct accumulation. Diabetologia 2004;47:1324-30.

45. Touboul PJ, Hennerici MG, Meairs S, et al. Mannheim carotid intima-media thickness and plaque consensus (2004-2006-2011). An update on behalf of the advisory board of the 3rd, 4th and 5th watching the risk symposia, at the 13th, 15th and 20th European Stroke Conferences, Mannheim, Germany, 2004, Brussels, Belgium, 2006, and Hamburg, Germany, 2011. Cerebrovasc Dis 2012;34:290-6.

46. Anon. Grading diabetic retinopathy from stereoscopic color fundus photographs - an extension of the modified Airlie House classification. ETDRS report number 10. Early Treatment Diabetic Retinopathy Study Research Group. Ophthalmology 1991;98(5 Suppl):786-806.

47. Hubbard LD, Brothers RJ, King WN, et al. Methods for evaluation of retinal microvascular abnormalities associated with hypertension/ sclerosis in the atherosclerosis risk in communities study. Ophthalmology 1999;106:2269-80.

48. Seino Y, Kuwata H, Yabe D. Incretin-based drugs for type 2 diabetes: focus on East Asian perspectives. J Diabetes Investig 2016;7(Suppl 1):102-9.

49. Ried-Larsen M, Thomsen RW, Berencsi K, et al. Implementation of interval walking training in patients with type 2 diabetes in Denmark: rationale, design, and baseline characteristics. Clin Epidemiol 2016:8:201-9.

50. Brinkløv CF, Thorsen IK, Karstoft K, et al. Criterion validity and reliability of a smartphone delivered sub-maximal fitness test for people with type 2 diabetes. BMC Sports Sci Med Rehabil 2016;8:31. 